\title{
Pollenallergie
}

\section{Lässt sich Blütenhonig zur Hyposensibilisierung nutzen?}

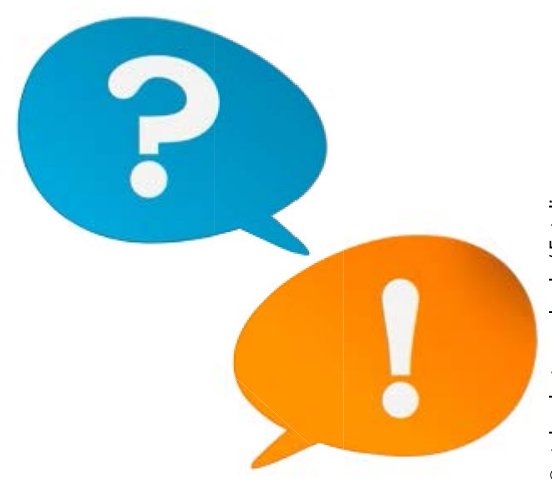

Frage: Natürlicher Blütenhonig enthält unvermeidbar geringe Anteile an Blütenpollen. Ist es vorstellbar, dass der Konsum von Blütenhonig vergleichbar einer SLIT wirkt? Gibt es Daten, ob, wie und ggf. in welchem Ausmaß regelmäßiger Konsum von Blütenhonig präventiv hinsichtlich einer Pollenallergie wirkt?

Antwort: Allergische Reaktionen gegen Honig stellen eine Rarität dar - auch bei Pollenallergikern [1]. Die wenigen berichteten Reaktionen reichen von einer Kontaktdermatitis [2] über ein Orales Allergie Syndrom (OAS) [3] bis zur Anaphylaxie $[4,5]$.

Man kann zwar davon ausgehen, dass auch einige Pollen im Honig vorhanden sind, diese Zahl dürfte jedoch in der Regel nicht ausreichen, um Symptome auszulösen. Bei den berichteten Fällen von Reaktionen auf Honig wird eine Kreuzreaktivität zwischen Korbblütler-Pollen und bestimmten Proteinen im Honig diskutiert [6]. Honig besteht im Übrigen aus einer heterogenen Mischung aus vorwiegend Zucker und Wasser, aber auch verschiedenen Proteinen.

Möglich ist auch, dass die seltenen allergischen Reaktionen auf Honig nicht durch Pollen, sondern durch geringe Reste von Insekten-Bestandteilen im
Honig hervorgerufen werden [7]. Dies wird als die häufigste Ursache einer Reaktion auf Honig angesehen $[8,9]$.

Bekannt ist darüber hinaus, dass oropharyngeale allergische Symptome (z. B. Juckreiz) auch ohne Genuss von Nahrungsmitteln direkt durch Pollenkontakt auf der Schleimhaut ausgelöst werden können.

Das Wirkprinzip der spezifischen Immuntherapie funktioniert über eine hohe Dosis, die das Immunsystem „überspielt“. Diese Dosen sind in der Regel höher als es durch eine natürliche Exposition erreicht wird. So werden bei der Pollen-Hyposensibilisierung 5 bis $20 \mu \mathrm{g}$ Allergen pro Erhaltungsdosis verabreicht. Im Honig werden solche therapeutischen Konzentrationen sicher nicht erreicht.

In der Literatur gibt es nur eine Studie, die die Frage eines möglichen therapeutischen Effektes untersucht hat. In dieser Studie wurde der Honig mit Pollen angereichert, wodurch man einen positiven Effekt gegenüber der Gruppe ohne Pollen-Honig-Therapie fand [10].

Zusammenfassend lässt sich feststellen, dass allergische Reaktionen auf Honig zwar beobachtet werden - aber selten. Sie sind entweder auf Pollen- oder wahrscheinlicher auf Insektenproteine zurückzuführen. Handelsüblicher Ho- nig ist nicht geeignet, eine spezifische Immuntherapie gegen Pollen zu unterstützen oder gar zu ersetzen.

\section{Literatur unter www.infectopharm.de}

Prof. Dr. med. Bodo Niggemann

Charité Centrum für Frauen-, Kinder- und Jugendmedizin mit Perinatalzentrum und Humangenetik, Klinik für Pädiatrie m. S. Pneumologie und Immunologie

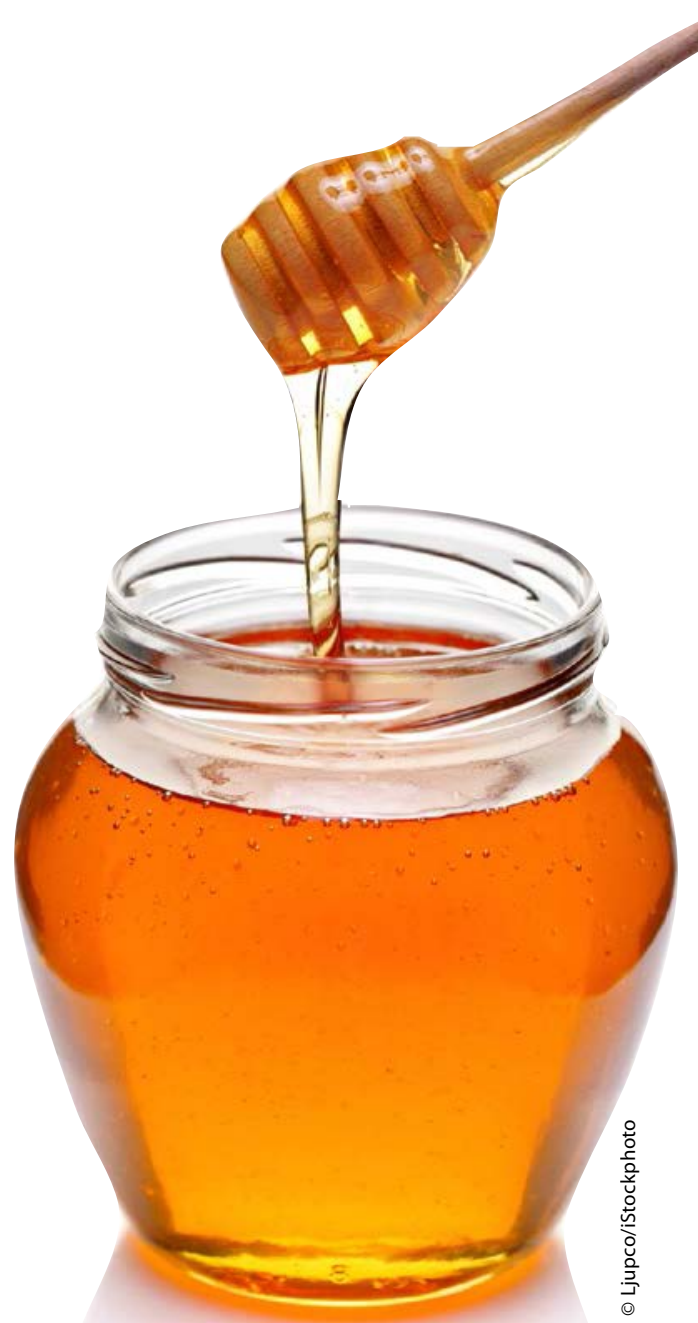

\section{Haben Sie auch eine fachliche Frage?}

Das „Consilium HNO“ ist ein Service des Unternehmens Infectopharm. Als HNO-Arzt haben Sie die Möglichkeit, selbst Fragen zum gesamten Fachgebiet zu stellen. Schreiben Sie dazu an Dr. Kristin Brendel-Walter, Von-Humboldt-Straße 1, 64646 Heppenheim, oder schicken Sie eine E-Mail an consilium@infectopharm.com. Ihre Frage wird anonym an ein Mitglied des Expertenpools weitergeleitet, Sie erhalten eine schriftliche Antwort.

Wenn Sie sehen wollen, was die Kolleginnen und Kollegen so fragen, können Sie im Archiv des "Consilium HNO" unter www.infectopharm.com/consilium stöbern. Außerdem werden redaktionell ausgewählte, allgemein interessante Anfragen zusätzlich in der Zeitschrift "HNO-Nachrichten“ publiziert. 